\title{
Nocebo and pain: adverse effects of excessive information
}

\author{
Nocebo e dor: os efeitos adversos do excesso de informação
}

Information to patients about their disease, especially about persistent pain, is important clinical practice approach. Improving (placebo) or worsening (nocebo) expectations may influence patients' evolution during treatment. The interest on this topic has increased the number of studies on behavioral and neurophysiologic bases of placebo and nocebo effects.

Placebo effect is related to a change in patients' clinical status attributed to an event, object or behavior in the therapeutic environment ${ }^{1}$. In general, placebo effect is always present in the clinical practice and may be defined as an inert effect within a positive context. If the context is negative, there is the opposite phenomenon, the nocebo effect, which creates negative expectations and worsens health status ${ }^{2}$. Nocebo effect may be defined as a set of events produced by negative expectations during therapeutic process ${ }^{2,3}$.

The word nocebo was created to define negative responses observed in placebo-treated groups ${ }^{4}$. Factors as environment (office, clinic, hospital), professional-patient relationship, verbal suggestions and patients' context (expectations, explicit memories, beliefs, emotions) may influence clinical results, thus cannot be ignored ${ }^{2,5}$.

Nocebo effect may increase pain intensity (hyperalgesia or induced allodynia), stress, anxiety, catastrophizing, in addition to increasing the search for health services, new therapeutic approaches, higher drug consumption and more surgeries to treat adverse effects produced by nocebo effect itself5. For example, excessive imaging exams for information about diagnosis and prognosis in chronic unspecific musculoskeletal conditions (such as low back pain, cervical pain, osteoarthritis) or disclosure of clinical results using medical jargons or specific technical terms, may negatively contribute and produce unnecessary attitudes and beliefs ${ }^{5}$. These approaches favor nocebo effect and may be considered iatrogenic, favoring worsening of pain, limitation of activities, development of negative expectations, anxiety, catastrophizing, avoidance and pain-related fear ${ }^{4}$.

Health education process itself may also contribute for the development of nocebo effect in people with persistent pain ${ }^{6}$. Nocebo responses may be manifested by negative verbal suggestions by professionals (such as in communicating diagnosis, therapeutic risks, prognosis and verbal interaction during treatment), by social learning (such as negative media campaigns) or by observation (experiences of others) ${ }^{3}$. Education models based exclusively on biomedical information contribute for nocebo effect development ${ }^{7}$. Maladaptive beliefs developed by patients may be exacerbated by poor professional-patient relationships, low heath services quality, by the media or even by individual experience and primarily include causal relations attributed to pain ${ }^{7,8}$. Pain amplification, as well as amplification of other emotional components present in the nocebo effect, seems to be related to the activation of brain affective-cognitive pathways ${ }^{8}$. Therapeutic ritual and psychosocial context involving treatment influence patients' brain activity and may produce better results by means of satisfaction and perceived effect ${ }^{1}$. It is suggested that attention be given to factors such as professional-patient relationship, communication, therapeutic techniques and treatment site ${ }^{5}$. This attention to contextual therapeutic process factors may help emphasizing placebo effect added to real therapy effect, in addition to decreasing nocebo effect ${ }^{3}$.

Predominant teaching-learning strategy in healthcare is still represented by professional-patient relationship verticalization, where patients are considered passive subjects unable to dialog with professionals to expose their beliefs and representations of their health status. This practice reflects authoritarianism in professional-patient relationship, denial of subjectivity of educative processes and lack of concern with the autonomy of patients as social subjects able to demand their interests. It is up to health professionals to use refined teaching-learning strategies based on scientific evidences, to dialog with patients about their health status and to give subsidies for the adoption of new health habits and approaches ${ }^{6,7}$. Professional-patient relationship horizontalization helps this interaction when recognizing patients as active subjects in the healthcare process ${ }^{5}$.

Quality and way of presenting contents may be a problem in the health education process ${ }^{3}$. Mass communication and social media might be important tools for the teaching-learning process, for being fast, economic and of wide access. However, quality of content and sources of information of such media are difficult to control. Information outside the therapeutic context such as negative campaigns (health warnings and modern concerns) may contribute for the nocebo effect developing maladaptive beliefs and favoring symptoms development ${ }^{8}$.

So, strategies are needed to maximize positive experiences related to the therapeutic process and to minimize those related to nocebo effect. It is important that the management of painful people go beyond the biomedical model (especially "pain=injury"). Health professionals should be alert and ready to optimize professional-patient relationship, to maximize comfort and environment where the treatment is performed. It is important to review the need and the moment to ask for new diagnostic exams to prevent unnecessary anxieties and concerns.

Professionals should have clinical information aligned with current and high quality scientific evidences. During the therapeutic process, emphasis should be given not to losses induced by pain, but rather to positive aspects, gradual therapy progressions and 
conquests, that is, focusing on functional aspects and not only on pain intensity ${ }^{8,9}$. Placebo and nocebo effects will always be present during the therapeutic process and may positively or negatively impact treatment effects (such as pain, incapacity, satisfaction, etc. $)^{5}$. So, it is up to us, health professionals, to make daily efforts in search for better therapeutic experiences for patients.

\section{Felipe Ribeiro Cabral Fagundes \\ Universidade Cidade de São Paulo, São Paulo SP, Brasil \\ E-mail: felipercfagundes@gmail.com}

Felipe J. J. Reis

Instituto Federal do Rio de Janeiro, Programa de Pós Graduação em Clínica Médica, Laboratório de Neuroimagem Avançada, Rio de Janeiro, Brasil E-mail: felipe.reis@ifrj.edu.br

Cristina Maria Nunes Cabral Universidade Cidade de São Paulo, São Paulo SP, Brasil E-mail: cristina.cabral@unicid.edu.br

\section{REFERÊNCIAS}

\footnotetext{
Benedetti F. Placebo and the new physiology of the doctor-patient relationship. Physiol Rev. 2013;93(3):1207-46.

Kaptchuk TJ, Miller FG. Placebo Effects in Medicine. N Engl J Med. 2015;373(1):8-9.

Benz LN, Flynn TW. Placebo, nocebo, and expectations: leveraging positive outcomes. J Orthop Sports Phys Ther. 2013;43(7):439-41.

Colloca L, Finniss D. Nocebo effects, patient-clinician communication, and therapeutic outcomes. JAMA. 2012;307(6):567-8.

Finniss DG, Kaptchuk TJ, Miller F, Benedetti F. Biological, clinical, and ethical advances of placebo effects. Lancet. 2010;375(9715):686-95.

Darlow B, Dowell A, Baxter GD, Mathieson F, Perry M, Dean S. The enduring impact of what clinicians say to people with low back pain. Ann Fam Med. 2013;11(6):527-34.

Benedetti F, Lanotte M, Lopiano L, Colloca L. When words are painful: unraveling the mechanisms of the nocebo effect. Neuroscience. 2007;147(2):260-71.

8. Marchand S, Gaumond I. Placebo and nocebo: how to enhance therapies and avoid unintended sabotage to pain treatment. Pain Manag. 2013;3(4):285-94.

9. Testa M, Rossettini G. Enhance placebo, avoid nocebo: How contextual factors affect physiotherapy outcomes. Man Ther. 2016;65-74.
} 